\title{
A methodological approach to logistics management in an inter- state confrontation
}

\author{
I.N. Kartsan",2,3,4,", A.O. Zhukov ${ }^{1,5,6,7}$, A.I. Bashkatov ${ }^{1}$, A.G. Kharlamov ${ }^{1}$, \\ S.A. Razzhivaykin ${ }^{1}$, E.D. Doronina ${ }^{1}$, M.A. Klementeva ${ }^{1}$ \\ ${ }^{1}$ FGBNU "Expert and Analytical Center", 33, Talalikhina Str., Building 4, Moscow, \\ Russia \\ ${ }^{2}$ Marine Hydrophysical Institute, Russian Academy of Sciences», 2, Kapitanskaya Str., \\ Sevastopol, Russia \\ ${ }^{3}$ Reshetnev Siberian State University of Science and Technology, 31, Krasnoyarsky \\ Rabochy Av., Krasnoyarsk, Russia \\ ${ }^{4}$ Sevastopol State University, University Str. 33, Sevastopol, Russia \\ ${ }^{5}$ Joint Stock Company "Special Research of Moscow Power Engineering Institute", 14 \\ Krasnokazarmennaya Str., Moscow, Russia \\ ${ }^{6}$ MIREA - Russian Technological University, 78, Vernadskogo Av., Moscow, Russia \\ ${ }^{7}$ Institute of Astronomy of the Russian Academy of Sciences, 48, Pyatnitskaya Str., \\ Moscow, Russia \\ *E-mail: kartsan2003@mail.ru
}

\begin{abstract}
To solve the formalized problem, the foundations of the methodology for assessing the need for state regulation of logistics support under non-military methods of power pressure, allowing to assess the need or redundancy of state influence on logistics support at the macro level, are proposed. This will improve the socio-economic component, for a positive effect in terms of increasing foreign trade turnover, as well as to increase employment and efficiency of human resources and lead to a reduction in the cost of production.
\end{abstract}

Keywords: transport logistics, sanctions, modelling, optimizations task 


\title{
Методологический подход к управлению логистикой в условиях межгосударственной конфронтации
}

\author{
И.Н. Карцан ${ }^{1,2,3,4, *}$, А.О. Жуков ${ }^{1,5,6,7}$, А.И. Башкатов ${ }^{1}$, А.Г. Харламов ${ }^{1}$, \\ С.А. Разживайкин ${ }^{1}$, Е.Д. Доронина ${ }^{1}$, М.А. Клементьева ${ }^{1}$ \\ ${ }^{1}$ ФГБНУ «Экспертно-аналитический центр», ул. Талалихина, 33/4, г. Москва, \\ Российская Федерация \\ ${ }^{2}$ ФГБУН ФИЦ «Морской гидрофизический институт РАН ул. Капитанская, 2, г. \\ Севастополь, Российская Федерация \\ ${ }^{3}$ ФГБОУ ВО «Сибирский государственный университет науки и технологий имени \\ академика М.Ф. Решетнева», просп. им. газ. «Красноярский рабочий», 31, г. \\ Красноярск, Российская Федерация \\ ${ }^{4}$ ФГАОУ ВО «Севастопольский государственный университет», ул. \\ Университетская, 33, г. Севастополь, Российская Федерация \\ ${ }^{5} \mathrm{AО}$ «ОКБ МЭИ», ул. Красноказарменная, 14, г. Москва, Российская Федерация \\ ${ }^{6}$ ФГБОУ ВО «МИРЭА - Российский технологический университет», ул. \\ Вернадского, 78, г. Москва, Российская Федерация \\ ${ }^{7}$ ФБУН «Институт астрономии Российской академии наук», ул. Пятницкая, \\ 48, г. Москва, Российская Федерация \\ *E-mail: kartsan2003@mail.ru
}

\begin{abstract}
Аннотация. Для решения формализованной задачи предложены основы методологии оценки необходимости государственного регулирования логистического обеспечения в условиях использования невоенных методов силового давления, позволяющие на макроуровне оценить необходимость или избыточность государственного влияния на логистическое обеспечение. Это позволит улучшить социально-экономическую составляющую, для положительного эффекта в части увеличения внешнеторгового оборота, а также для увеличения занятости и эффективности использования трудовых ресурсов и приведет к снижению себестоимости выпускаемой продукции.
\end{abstract}

Ключевые слова: транспортная логистика, санкции, моделирование, оптимизация задача

\section{1. Введение}

Современные геополитические разногласия обусловливают применяемые к России невоенные методы воздействия, заключающиеся в санкционном давлении. Это оказывает воздействие на развитие экономических систем и влияет на эффективность производственных и транспортно-логистических цепочек, значительно увеличивая цену оказания подобного рода услуг.

Приведенные вызовы и условия новы для отечественной логистической науки - это во многом обусловливает необходимость разработки или адаптации научно-методического 
аппарата для оценки эффективности производственных и транспортно-логистических цепочек в складывающихся условиях. При этом под оценкой эффективности производственных и транспортно-логистических цепочек понимается теория и практика эффективного управления материальными и сопутствующими им информационными и сервисными потоками. Под материальными потоками при этом понимаются различные материальные ценности, рассматриваемые в процессе приложения к ним логистических операций.

Для оценки эффективности логистического управления в монографии рассматриваются глобальные проблемы управления материальными потоками, то есть макроуровень отношений. Задачей логистики в данных условиях является планирование материальных потоков, координация действий участников движения, управление и контроль всех стадий, обеспечение движения и преобразования.

Оценку эффективности системы логистического обеспечения в условиях межгосударственной конфронтации с использованием невоенных методов силового давления предлагается проводить с учетом совокупности макроэкономических параметров социально-экономического развития государства. Это позволит на системном уровне оценивать эффективность производственных и транспортно-логистических цепочек в заданных условиях. Для решения приведенной задачи необходима ее математическая формализация.

В условиях, применяемых к России невоенных методов воздействия, преимущественно заключающиеся в санкционном давлении, для обеспечения конкурентоспособности транспортно-логистических услуг необходимым является государственное регулирование логистического обеспечения с учетом совокупности макроэкономических параметров социально-экономического развития [1].

\section{2. Методологический подход}

Замысел государственного регулирования и поддержки логистического обеспечения в данных условиях заключается в формировании функциональных элементов и объединении их в систему $[2,3]$. Это позволит на системном уровне оценить эффективность логистического обеспечения и необходимость государственного вмешательства посредством реализации предлагаемых этапов методологии:

- моделирование влияния логистического обеспечения на социальноэкономическое развитие в условиях межгосударственной конфронтации с использованием невоенных методов силового давления.; 
- подготовка необходимой информации для моделирования влияния логистического обеспечения на социально-экономическое развитие в условиях межгосударственной конфронтации с использованием невоенных методов силового давления.;

- решение оптимизационной задачи оценки влияния логистического обеспечения на социально-экономическое развитие в условиях межгосударственной конфронтации с использованием невоенных методов силового давления.

Рассмотрим более подробно каждый из предлагаемых этапов.

\section{1. Моделирование}

Моделирование влияния логистического обеспечения на социально-экономическое развитие в условиях межгосударственной конфронтации с использованием невоенных методов силового давления.

Моделирование как метод приобретения информации о работе транспортнологистических цепочек следует сравнить с математическим анализом, с одной стороны, и с экспериментальным испытанием, с другой. При этом необходимо отметить, что анализ требует привлечения меньшего количества ресурсов, чем моделирование, которое, в свою очередь, требует меньшего объема ресурсов, чем практические испытания.

Моделирование занимает промежуточное положение между анализом и экспериментальным испытанием. Значение этих двух этапов проектирования не следует преуменьшать, так как они являются основой методического аппарата при проектировании систем. В настоящее время ни одна транспортно-логистическая система не будет создаваться (модернизироваться) без какого-либо моделирования [4].

Для оценки влияния логистического обеспечения на социально-экономическое развитие в заданных условиях, необходимо сформировать (пусть даже в крупную клетку) предмет и цель исследования.

Предмет - логистическое обеспечение в условиях межгосударственной конфронтации с использованием невоенных методов силового давления.

Цель - оценка влияния логистического обеспечения на социально-экономическое развитие государства (макроуровень).

Для этого необходимо в рассматриваемом социально-экономическом процессе выделить структурные и функциональные элементы, соответствующие приведенной цели, при этом выделяются наиболее важные качественные характеристики этих элементов. Словесно описывается взаимосвязь между элементами взаимовлияния. Вводятся символические обозначения для учитываемых характеристик предмета исследования. 
В основу построения математической схемы взаимовлияния была положена идея синтеза моделей Г.Х. Гуда, Р.Э. Макола [5], формализованная через динамическую форму Лотки-Вальтера [6]. В результате была синтезирована ориентировочная форма модели взаимовлияния социально-экономического и логистического развития, которая, в свою очередь, может учитывать дополнительные параметры. Таким образом, предложена модель, формализованная в виде системы обыкновенных дифференциальных уравнений:

$$
\left\{\begin{array}{l}
\frac{d x}{d t}= \pm a_{1} x \pm a_{2} x y \pm a_{3} x w \\
\frac{d y}{d t}= \pm b_{1} y \pm b_{2} y x \pm b_{3} y w \\
\frac{d w}{d t}= \pm c_{1} w \pm c_{2} w x \pm c_{3} w y \\
x(0)=x_{0}, y(0)=y_{0}, w(0)=w_{0}
\end{array}\right.
$$

где $x$-переменная, характеризующая социальную составляющую (численность населения);

$y$ - переменная, характеризующая экономическую составляющую (ВВП государства);

$w$ - переменная, характеризующая транспортно-логистическую составляющую (стоимость логистического обеспечения);

$a_{1}$ - коэффициент естественных демографических процессов;

$a_{2}$ - коэффициент влияния экономической составляющей на социальную;

$a_{3}$-коэффициент влияния транспортно-логистического обеспечения

на социальную составляющую;

$b_{1}$ - коэффициент естественных экономических процессов;

$b_{2}$ - коэффициент влияния социальной составляющей на экономическую;

$b_{3}$ - коэффициент влияния транспортно-логистического обеспечения

на экономическую составляющую;

$c_{1}$ - коэффициент, характеризующий транспортно-логистическое обеспечение;

$c_{2}$ - коэффициент влияния социальной составляющей на транспортно-логистическую;

c3 - коэффициент влияния экономической составляющей на транспортно-логистическую.

Таким образом, предложена математическая форма модели взаимовлияния социальной, экономической и военной систем государства в виде системы обыкновенных дифференциальных уравнений, что позволяет отследить, спрогнозировать критические отклонения рассматриваемых параметров для принятия решения о государственном вмешательстве. 


\section{2. Подготовка информации для моделирования}

Подготовка необходимой информации для моделирования влияния логистического обеспечения на социально-экономическое развитие в условиях межгосударственной конфронтации с использованием невоенных методов силового давления

Форма приведенной модели обусловливает формализацию регрессионного анализа, учитывающего корреляцию рассматриваемых параметров. Для этого предложено использовать классические подходы регрессионного анализа оценки коэффициентов, характеризующих связь и влияние транспортно-логистических цепочек на социальноэкономическое развитие государства.

Учитывая то, что рассматривается мирное время (без скачкообразного изменения), допустимо рассматривать коэффициенты, характеризующие общую динамику посредством разложения в ряд Тейлора [7]. Для примера приведено разложение от одной переменной:

$$
l_{i}=K_{i 0}+K_{i}\left(\chi_{i} B_{i}-B_{i 0}\right)
$$

где $l_{i}$ - относительный коэффициент влияния на приращение начальных значений точки линейной аппроксимации;

$K_{i 0}$ - начальное значение коэффициента $l_{i}$ в начальной точке;

$K_{i}$ - производная коэффициента;

$\chi_{i}$ - доля относительно $B_{i}$;

$B_{i}$ - системообразующий показатель;

$B_{i 0}-$ задаваемый частный показатель.

Представленный вид соответствует виду метода регрессии - наименьших квадратов. Поэтому анализ достаточности и корректности исходных данных ввиду того, что используется аппроксимация линейного типа, предложено осуществлять посредством использования фундаментального уравнения дисперсионного анализа [8]:

$$
\sum\left(A_{i}-\bar{A}_{i}\right)^{2}=\sum\left(A_{i}-\hat{A}_{i}\right)^{2}+\sum\left(\hat{A}_{i}-\bar{A}_{i}\right)^{2}
$$

где $\quad A_{i}-\bar{A}_{i}-$ отклонение $i$-го наблюдения от общего среднего;

$A_{i}-\hat{A}_{i}-$ отклонение $i$-го наблюдения от его предсказанного или вычисленного значения; 
$\hat{A}_{i}-\bar{A}_{i}-$ отклонение предсказанного значения $i$-го наблюдения от среднего.

Для практического применения допустима следующая формализация:

$$
R^{2}=\frac{\sum_{i}^{n}\left(\hat{A}_{i}-\bar{A}_{i}\right)^{2}}{\sum_{i}^{n}\left(A_{i}-\bar{A}_{i}\right)^{2}}\left\{\begin{array}{l}
R^{2} \rightarrow 1-\text { данные корректны } \\
R \rightarrow 0-\text { данные некорректн bl }
\end{array}\right.
$$

В том случае, если $R^{2}=0 . .0,4$, то исходные данные нельзя считать корректными, для их корректировки (дополнения) предложено использовать экспертный метод $[4,8]$.

Таким образом, рассмотрен принцип оценки достаточности и корректности исходной информации для оценки эффективности производственных и транспортнологистических цепочек в условиях межгосударственной конфронтации с использованием невоенных методов силового давления.

\section{1. Решение задачи}

Решение оптимизационной задачи оценки влияния логистического обеспечения на социально-экономическое развитие в условиях межгосударственной конфронтации с использованием невоенных методов силового давления.

Нахождение требуемого влияния логистического обеспечения на социальноэкономическое обеспечение предлагается посредством применения модернизированного метода Ньютона [5].

Необходимым условием реализации метода Ньютона является наличие аналитической формы, которая позволяет получить точные алгоритмы вычисления производных. Суть метода заключается в следующем.

Выбирается начальный вектор параметров $\left\{\theta_{i(n)}\right\}$, по которым будет проводиться оптимизация. Строится алгоритм, по которому определяется следующий вектор выбранных параметров, который является новым приближением к оптимальному значению $\left\{\theta_{i(n+1)}\right\}$.

В качестве такого преобразования для метода Ньютона используется следующее выражение:

$$
\theta_{i(n)}=\theta_{1(n)}-\alpha \cdot\left|f_{\theta(n)}^{\prime \prime}\right|^{-1} \times\left|f_{\theta(n)}^{\prime}\right|
$$

где: $\theta_{(n+1)}=\left|\begin{array}{c}\theta_{1(n+1)} \\ \ldots \\ \theta_{N(n+1)}\end{array}\right|-$ вектор новых значений параметров; 
$\theta_{1(n)}=\left|\begin{array}{c}\theta_{1(n)} \\ \ldots \\ \theta_{N(n+1)}\end{array}\right|-$ вектор предыдущих параметров;

$\left|f_{\theta(n)}^{\prime \prime}\right|=\left|\begin{array}{ccc}f_{\theta_{1}(n)}^{\prime \prime} & \ldots & f_{\theta_{1} \theta_{M}(n)}^{\prime \prime} \\ \ldots & \ldots & \ldots \\ f_{\theta_{N} \theta_{1}(n)}^{\prime \prime} & \ldots & f_{\theta_{1} \theta_{M}(n)}^{\prime \prime}\end{array}\right|$ - матрица частных производных, вычисляемых для предыдущих параметров;

$\left|f_{\theta(n)}^{\prime}\right|=\left|\begin{array}{c}f_{1(n)}^{\prime \prime} \\ \ldots \\ f_{\theta_{N}(n)}^{\prime \prime}\end{array}\right|-$ вектор первых производных для предыдущих параметров;

$\alpha$ - специально подбираемая регуляризирующая функция.

Алгоритм данного метода основан на квадратичной аппроксимации, такая аппроксимация, оставаясь достаточно простой, в то же время является намного более точной, чем линейная, используемая в классических градиентных методах, что позволяет строить на ее основе эффективные алгоритмы.

\section{3. Заключение}

Таким образом, предложены основы методологии для оценки необходимости государственного регулирования логистического обеспечения в условиях использования невоенных методов силового давления, позволяющие на макроуровне оценить необходимость или избыточность государственного влияния на логистическое обеспечение. Это позволит улучшить социально-экономическую составляющую, для положительного эффекта в части увеличения ВВП и внешнеторгового оборота, а также для увеличения занятости и эффективности использования трудовых ресурсов; будет способствовать снижению себестоимости выпускаемой продукции. Также адаптирована методика регрессионного анализа статистической информации для расчета коэффициентов системы уравнений, описывающей влияние логистичсеских расходов на социальноэкономическое развитие государства, отличающаяся уникальной адаптацией к рассматриваемой системе дифференциальных уравнений.

\section{Благодарности}

Работа выполнена в рамках государственного задания Минобрнауки России по теме «Концептуальное моделирование информационно-образовательной среды воспроизводства человеческого капитала в условиях цифровой экономики» (Шифр FNRN - Е). Работа выполнена в рамках государственного задания Минобрнауки России по теме «Разработка новых методов автономной навигации космических аппаратов в космическом 


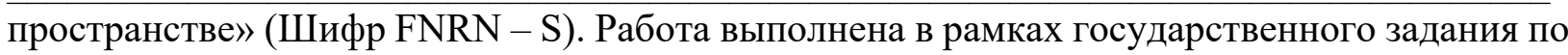
теме № 0555-2021-0005.

\section{Список литературы}

[1] Носов, А.Л. Методология управления развитием инфраструктуры региональной логистики, диссертация док. эк. наук. / А.Л. Носов. - Санкт-Петербург, 2007, - 310 c.

[2] Маккол, Р. Справочник по системотехнике / Пер. с англ.; под ред. А.В. Шиленко. / Р. Маккол. - М.: «Советское радио», 1970. - 688 с.

[3] Пестун, У.А. Управление социальной и экономической системами в условиях требуемого уровня обороноспособности / У.А. Пестун, А.О. Жуков. - М.: ФГБУН «Аналитический центр» Минобрнауки России, 2017. - 144 с.

[4] Буренок, В.М. Математические методы и модели в теории информационноизмерительных систем / В.М. Буренок, В.Г. Найденов, В.И. Поляков. - М.: Машиностроение, 2011. - 334 с.

[5] Гуд, Г. Системотехника: введение в проектирование больших систем / Г. Гуд, Р. Маккол. - М.: Советское радио, 1962. - 383 с.

[6] Жуков, А.О. Основы экспертного оценивания / А.О. Жуков, У.А. Пестун. - М.: ФГБУН «Аналитический центр» Минобрнауки России, 2017. - 65 с.

[7] Химмельблау, Д. Прикладное нелинейное программирование / Д. Химмельблау. М.: Мир, 1975. - 536 c.

[8] Карасева, М.В. Метапоисковая мультилингвистическа система / М.В. Карасева, И.Н. Карцан, П.В. Зеленков // Вестник Сибирского государственного аэрокосмического университета им. академика М.Ф. Решетнева. - 2007. - № 3(16). - С. 69-70. 